\title{
PIXE Analysis of NIST and IAEA Biological Reference Materials
}

\author{
${ }^{1}$ B. Gowri Naidu; ${ }^{* 2}$ P. Sarita $\&{ }^{3}$ G. J. Naga Raju \\ ${ }^{1}$ Research Scholar, Department of Electronics and Physics, GIS, GITAM (Deemed to be University), Visakhapatnam (India) \\ ${ }^{* 2}$ Associate Professor, Department of Physics, GIT, GITAM (Deemed to be University), Visakhapatnam (India) \\ ${ }_{3}^{3}$ Assistant Professor, Department of Physics, UCEV-JNTUK, Vizianagaram (India)
}

\begin{tabular}{|c|c|}
\hline ARTICLE DETAILS & ABSTRACT \\
\hline $\begin{array}{l}\text { Article History } \\
\text { Published Online: } 05 \text { July } 2018\end{array}$ & \multirow{3}{*}{$\begin{array}{l}\text { Particle induced X-ray emission (PIXE) technique was employed for analysis of trace } \\
\text { elements in two biological standard reference materials: Bovine Liver (1577c) and Animal } \\
\text { Blood (A-13) prepared by National Institute of Standards and Technology (NIST), USA and } \\
\text { International Atomic Energy Agency (IAEA), Vienna respectively. The PIXE experiments } \\
\text { were carried out by using } 3 \mathrm{MV} \text { Tandem Pelletron Accelerator facility at lon Beam } \\
\text { Laboratory (IBL), Institute of Physics (IOP), Bhubaneswar, India. A proton beam of } 3 \mathrm{MeV} \\
\text { energy was used to excite the samples under study. The concentrations of the elements } \\
\text { obtained in the reference materials were compared with the certified values of NIST and } \\
\text { IAEA. The determined values were } 88-125 \% \text { relative to NIST and IAEA certified values } \\
\text { except for Mn in the Animal Blood sample. }\end{array}$} \\
\hline $\begin{array}{l}\text { Keywords } \\
\text { PIXE, Trace elements, Reference } \\
\text { materials, Bovine Liver, Animal Blood }\end{array}$ & \\
\hline $\begin{array}{l}\text { "Corresponding Author } \\
\text { Email: sarita.padala[at]gitam.edu }\end{array}$ & \\
\hline
\end{tabular}

\section{Introduction}

Several analytical methods like X-ray Fluorescence (XRF) [1, 2], Particle Induced X-ray Emission (PIXE) [3], Neutron Activation Analysis (NAA) [4], Atomic Absorption Spectroscopy (AAS) [5], Inductively Coupled Plasma Mass Spectroscopy (ICP-AES) [6], Inductively Coupled Plasma Atomic Emission Spectroscopy (ICP-MS) [7], have been employed to identify and quantify trace elements in a wide variety of samples including biological samples. Among these analytical methods, AAS, ICPAES, ICP-MS are routinely used for the analysis of samples in a destructive way for which the analyte should be in the form of liquid. In order to overcome the loss of volatile elements and minimize the contamination during the digestion procedure, non-destructive analytical techniques such as XRF, PIXE, NAA etc. are preferred for the biological samples. PIXE is a multielemental nuclear analytical technique and it permits analysis of minute samples lesser than a milligram without any complex sample preparation. It permits simultaneous analysis of elements from $\mathrm{Na}$ to $\mathrm{U}$ in a short time and facilitates detection of elements at ppm to sub-ppb level. In view of these advantages, PIXE is universally accepted as a reliable method for elemental analysis in several fields [8-11].

Comparison of values determined by PIXE with certified reference values is important in order to know the uncertainty associated with instrumental parameters and to validate the technique. In the present study, elemental concentrations determined by PIXE in National Institute of Standards and Technology (NIST) standard reference material (SRM)-Bovine Liver (1577c) and International Atomic Energy Agency (IAEA) reference material $(\mathrm{RM})$-Animal Blood ( $\mathrm{A}-13)$ were compared with the certified values.

\section{Materials and Methods}

\subsection{Samples}

Freeze-dried and powdered samples of SRM Bovine Liver (1577c) and RM Animal Blood (A-13) were procured from NIST and IAEA respectively.

\subsection{Sample preparation}

The NIST SRM-Bovine Liver (1577c) and IAEA RM-Animal Blood (A-13) were carefully weighed using a sensitive analytical balance. Sample weighing $90 \mathrm{mg}$ was taken and mixed with 60 $\mathrm{mg}$ of high purity graphite powder in an agate mortar homogeneously. The resulting sample weighing $150 \mathrm{mg}$ was pressed as a pellet with dimensions of $12 \mathrm{~mm}$ diameter and 2 $\mathrm{mm}$ thickness using $\mathrm{KBr}$ Hydraulic Press. Five such pellets were prepared for each reference material. These pellets were used as targets for PIXE experiments.

\subsection{Experimental details}

\subsubsection{Particle Induced $X$-ray Emission (PIXE)}

Particle Induced X-ray emission (PIXE) was first demonstrated by Johansson et al. in 1970 at the Lund Institute of Technology [12]. PIXE analytic technique consists of two parts. The first is to identify the elements present in the sample target from energies of the characteristic peaks in the X-ray emission spectrum and second part is to determine the quantity of the elements present in the target from the intensity of their characteristic peaks. The detailed PIXE instrumentation used in the present study is as follows.

\subsubsection{Tandem Pelletron Accelerator}

The accelerator facility used at IBL, IOP, Bhubaneswar consists of a $3 \mathrm{MV}$ Tandem Pelletron Accelerator (National Electrostatic Corporation, U.S.A., model 9SDH-2). The SNICS ion source, i.e., the source of negative ions from cesium sputtering, was used for generating protons. The cesium vapour stream formed by heating solid/liquid cesium was ionised, forming positively charged cesium ions which were then accelerated to the tip of the cathode. Cesium ions sputtered the cold cathode material made of pressed $\mathrm{TiH} 2$ powder. The sputtered hydrogen atoms were ionised and then accelerated through an aperture in the ioniser. On entering the accelerator the beam of negative ions was accelerated towards a positively charged terminal at the centre of the accelerator tube. The accelerator column surrounding the tube maintained an even 
voltage gradient up along the tube to the terminal. The terminal can hold a maximum of $3 \mathrm{MV}$. The charge was delivered to the terminal by two nylon chains containing steel pellets on each link. The electrons were stripped from the beam at mid terminal by the introduction of a small amount of nitrogen gas to the vacuum in the terminal space. Collisions between ion beam and nitrogen gas molecules produced neutral as well as single and multiple charged positive ions. The fast moving positively charged beam particles then see the ground potential at the far end of the accelerator as a further attractive force and continue to be accelerated until their exit from the accelerator. The electric insulation of the high voltage terminal was achieved by filling SF6 gas in the tank space surrounding the accelerator tube and maintaining it about $65-70$ psi pressure. A switching magnet was used to steer the ions of the desired charge into the different beam lines. The energy analysis, focusing and deflection of the beam were all obtained by means of magnetic elements (dipoles and quadrupoles). The energy of the beam at the target is a function of the pre-acceleration stage (cathode, extractor, and bias voltages), the terminal voltage of the accelerator, and the charge of the ion selected by the switching magnet. Using a magnetic quadrupole lens further steering was employed to bring a suitably focused ion beam onto the target in the target chamber.

\subsubsection{Scattering chamber}

A scattering chamber is one of the essential parts in accelerator based experiments. The scattering chamber is octagonal in shape with 20" diameter and 15" height and consists of several ports for X-ray and $\gamma$-ray detectors. In order to detect X-rays, a Si (Li) detector (ORTEC, Model No. SLP06165-OPT-0-5) was kept in one of the ports in such a way that it was close enough to the target to provide a large solid angle. A viewing window provided visual indication of the size and location of the beam spot on target and thereby facilitated the focussing of the beam exactly onto the target. The target was placed at an angle of 450 to the beam direction and the $\mathrm{Si}(\mathrm{Li})$ detector was placed at an angle of 450 to the target position. The chamber was usually maintained at low vacuum $\left(\sim 10^{-6}\right.$ torr) during the measurements by using rotary and diffusion pumps. Since the thick targets were electrically conductive, the beam current was measured and integrated directly on the target. In the present work, the charge integrator was coupled directly to the target holder in order to monitor the beam current.

\subsubsection{Electronics and Data Acquisition Systems}

The characteristic $X$-rays emitted from various samples as a consequence of the ion-atom collision processes were detected by the $\mathrm{Si}(\mathrm{Li})$ detector. The X-rays before reaching the active volume of the detector passed through a $95 \mu \mathrm{m}$ metallized Mylar chamber window, a $30 \mathrm{~mm}$ air gap and a $12.7 \mu \mathrm{m} \mathrm{Be}$ window of detector. The total distance from the target to $\mathrm{Si}(\mathrm{Li})$ detector (active area is $12.5 \mathrm{~mm}^{2}$ ) was $62 \mathrm{~mm}$. A negative bias of $450 \mathrm{~V}$ was applied to the detector from the ORTEC power supply (Model 459). After passing through the Mylar window of the scattering chamber, the characteristic X-rays were detected. An aluminium aperture of known dimensions was placed in front of the $\mathrm{Si}(\mathrm{Li})$ detector for an accurate determination of the solid angle subtended by the detector at the beam position on the sample surface. The signals from the detector preamplifier were processed with an ORTEC spectroscopic amplifier (Model 572), enabling pile-up rejection, and then fed into an Analog to Digital Converter. Data manipulation and storage were performed with a Canberra S100 counting system, based on an IBM personal computer. Spectral data was then transferred to a computer where further data analyses were carried out. An ORTEC-448 research pulsar was connected to the counting system for monitoring the stability of the spectrum.

\subsection{Si (Li) Detector}

The detector system consists of a cooled lithium drifted silicon crystal, cooled field effect transistor (FET), high gain and low noise preamplifier, cryostat with a Be window, liquid nitrogen $\left(\mathrm{LN}_{2}\right)$ dewar, and cable pack. The detector has 12.5 $\mathrm{mm}^{2}$ active area and $12.7 \mu \mathrm{m}$ thick Be window. The SLP series preamplifier is capable of an energy rate performance in excess of $750 \mathrm{MHz}$. If the $\mathrm{LN}_{2}$ supply is exhausted and the detector begins to warm up while high voltage is applied, the high voltage is automatically shut off, thus protecting the FET from damage.

\subsection{Si (Li) Role in PIXE Experiments}

$\mathrm{K}$ X-rays of high ' $Z$ ' elements would be properly detected by using HPGe or $\mathrm{Ge}(\mathrm{Li})$ detectors because they continue to have the maximum efficiency up to $100 \mathrm{keV}$. However, K X-ray crosssection for protons rapidly falls as ' $Z$ ' increases. This renders the use of $\mathrm{K} X$-rays of high $Z$ elements rare in PIXE by using $\mathrm{Ge}$ detectors. Instead of K X-rays of the high Z elements, L X-rays are used, since they are well matched to the range of maximum efficiency of $\mathrm{Si}(\mathrm{Li})$ detector which is typically 2 to $20 \mathrm{keV}$. This is why lower atomic number elements $(Z<50)$ are identified by their corresponding $\mathrm{K} \mathrm{X}$-ray energies while the higher atomic number elements are identified by their $L X$-ray energies in a typical PIXE spectrum obtained from a muti-element specimen. Further, the $\mathrm{Si}(\mathrm{Li})$ detector records the spectrum of all detectable elements at a single setting of the instrument and in a single measurement.

\subsubsection{Energy Calibration}

PIXE spectrum is very complex having fairly crowded mixture of $\mathrm{K} X$-ray lines and $\mathrm{L} X$-ray lines. Identification of each element present in the PIXE spectrum depends on the accurate energy calibration of the detector. Present $\mathrm{Si}(\mathrm{Li})$ detector was calibrated by using radioactive sources ${ }^{241} \mathrm{Am}$ and ${ }^{55} \mathrm{Fe}$ supplied by International Atomic Energy Agency (IAEA). The K X-ray and $\mathrm{L}$ X-ray components of above mentioned isotopes were well resolved and the centroid of each photo peak was determined by the following relationship

$$
\text { and } \quad \begin{aligned}
& \mathrm{X}_{\mathrm{c}}=\sum(\mathrm{Xi} . \mathrm{Yi}) / \sum \mathrm{Yi} \\
& \mathrm{Y}_{\mathrm{c}}=\sum(\mathrm{Xi} . \mathrm{Yi}) / \sum \mathrm{Xi}
\end{aligned}
$$

where $X_{c}$ and $Y_{c}$ are the coordinates of the centroid, $X_{i}$ is the channel number and $Y_{i}$ is the corresponding count rate. After subtracting the background, the net count rate was obtained. After getting the centroid of each X-ray line, the channel number of each photo peak centroid was plotted against the energy of the photo peak. The operations like background subtraction and 
determination of the peak centroid were performed by using GUPIXWIN software package.

\subsubsection{Resolution Characteristics of the Detector System}

Resolution of a detector is an important parameter in the analysis of X-ray spectra particularly L X-ray spectra. The resolution of solid state detector is expressed in terms of the full width at half maximum (FWHM) of a standard line. The resolution of the present $\mathrm{Si}(\mathrm{Li})$ detector was measured at $5.9 \mathrm{keV} \mathrm{K} \mathrm{X-ray} \mathrm{energy} \mathrm{of}{ }^{55} \mathrm{Mn}$. The standard Fe-55 source was kept at a distance of $5 \mathrm{~cm}$ from the detector window, so as to ensure that the dead time was of the order of $1 \%$. The $\mathrm{K}$ X-ray spectrum with Fe-55 source was recorded over a long time to obtain good statistics. From the resulting spectrum the FWHM was determined at an optimum time constant of the amplifier. The resolution of the $\mathrm{Si}(\mathrm{Li})$ detector employed in the present work was found to be $160 \mathrm{eV}$ at $5.9 \mathrm{keV}$ energy.

\subsubsection{Efficiency of the Si(Li) Detector}

An accurate efficiency calibration of a $\mathrm{Si}(\mathrm{Li})$ detector is necessary for both fundamental and applied research. The present trace elemental analysis was mainly concerned with the measurement of low energy X-rays (1-20 keV) emitted from the sample due to proton bombardment and hence for this purpose, a high resolution $\mathrm{Si}(\mathrm{Li})$ detector was employed. The counting efficiency of a detector is a fundamental quantity in the detection process. The intrinsic efficiency of a detector is defined as the number of pulses recorded out of the number of radiation quanta incident on detector. Because of the presence an aperture in front of the detector it subtends a solid angle $\Omega$ at the beam spot on the surface of the sample which can be estimated with greater accuracy. A factor, $\Omega / 4 \pi$, of total photons emitted from the process is seen by the detector. In addition to the intrinsic efficiency of the detector, geometrical factor $\Omega / 4 \pi$ is included. Thus, the intrinsic efficiency of the detector is given by sint $=\varepsilon / \varepsilon g e o$, where $\varepsilon$ is the total efficiency of detection of the Xrays and $\varepsilon g e o$ is the geometrical efficiency which depends on the geometrical arrangement of the experiment. The total efficiency of the detector is the product of the intrinsic efficiency and geometrical efficiency. Theoretically, the intrinsic efficiency of $\mathrm{Si}(\mathrm{Li})$ detector is estimated from the detector parameters as supplied by the manufacturer. The following expression is used to calculate the intrinsic efficiency $\varepsilon d$, as illustrated by Padhi [12].

$$
\varepsilon d(E)=e-\left(\mu_{B e} \cdot X_{B e}+\mu_{A u} \cdot X_{A u}+\mu_{S i} \cdot \Delta X_{S i}\right) .\left(1-e-\mu_{S i} X_{S i}\right)
$$

where $\mu$ 's represent the absorption coefficients due to Be window, Au layer on the Silicon crystal and $\mathrm{Si}(\mathrm{Li})$ crystal at the $X$-ray energy $E, \Delta x S i$ is the thickness of the insensitive region of the $\mathrm{Si}(\mathrm{Li})$ crystal. The values of $X$ were specified by the manufacturer. The absorption coefficients were calculated by using the computer code XCOM [13].

The total efficiency of the spectrometer, consisting of the intrinsic efficiency of the $\mathrm{Si}(\mathrm{Li})$ detector, the detector solid angle and the X-ray attenuation factor for absorption between the target and the intrinsic region of the detector, was determined by the method described in detail by Pajek et al.[14]. The literature abounds with data on K-shell ionization cross-sections by protons [15-17], which generally show good agreement (i.e., within the claimed experimental uncertainties) with the K-shell ionization data of the ECPSSR theory. Efficiency of the detector in the present work was calculated by substituting the K-shell ionization cross-sections instead of $\mathrm{K} X$-ray production cross-sections for the elements $\mathrm{Ca}, \mathrm{V}, \mathrm{Fe}, \mathrm{Cu}$ and $\mathrm{Zn}$ by using $2.5 \mathrm{MeV}$ protons. Theoretical ionization cross sections were taken from the ISICS code [18]. The experimental detector efficiency was calculated using the formula

$$
\varepsilon_{d}=\frac{4 \Pi Y_{x} \sigma_{R}(\theta) \Delta \Omega_{p}}{\varepsilon_{t} \varepsilon_{a} Y_{R} \Delta \Omega_{x} \sigma_{K}^{x}}\left(\frac{t_{x}}{t_{R}}\right)
$$

where,

$\sigma_{\mathrm{K}}^{\mathrm{X}}=\mathrm{K}$ Xray theoretical production ionizations from ISICS code $\mathrm{Y}_{\mathrm{X}}(\theta)=$ Measured $\mathrm{KX}$ - ray yield

$\mathrm{Y}_{\mathrm{R}}(\theta)=$ Measured Rutherford yield

$\sigma_{\mathbb{R}}(\theta)=$ Differential Rutherford scattering cross - section

$\varepsilon_{d}=\operatorname{Si}($ Li) detector intrinsic efficiency

$\varepsilon_{a}=$ Absorption correction for Mylar chamberwindow and aîr path

$\varepsilon_{t}=$ Correction due to self absorption of the target thickness $\Delta \Omega_{p}=$ Solid angle subtended by the charged particle silicon surface barrier detector

$\Delta \Omega_{x}=$ Solid angle subtended by the $\mathrm{Si}(\mathrm{Li}) \mathrm{X}$ - ray detector

$t_{x}=$ Dead time correction for $X-$ ray counting

$t_{R}=$ dead time correction for charged particle counting

\subsection{Data Analysis}

The spectrum recorded in a PIXE measurement with 2-3 $\mathrm{MeV}$ protons is a sum of contributions of the different elements present in the target irradiated: $\mathrm{K} X$-rays from the lighter elements, $K$ and $L X$-rays for the medium- $Z$ elements, $M$ and $L X$-rays for the higher-Z ones. Each of the elements of low and medium atomic number contributes about six distinct $\mathrm{K} X$-ray lines and those of high atomic number contribute about twenty $\mathrm{L}$ X-ray lines. The peaks, approximately Gaussian in shape, are superimposed on a continuous background originating from secondary bremsstrahlung and Compton scattering of $y$-rays also produced in the interactions. The resulting $\mathrm{Si}(\mathrm{Li})$ spectrum is therefore complex and peak overlaps occur frequently [19]. The $\mathrm{K}_{\beta} \mathrm{X}$-ray component of an element with atomic number $\mathrm{Z}$ may overlap with the $\mathrm{K}_{\alpha} \mathrm{X}$-ray component of the element with atomic number $Z+1$. Similarly the $L_{\alpha} X$-ray component of a high $Z$ element may overlap with the $\mathrm{K}_{\alpha} \mathrm{X}$-ray component of a low $\mathrm{Z}$ element. In order to compute the intensities of different $X$-ray lines from overlapped PIXE spectra a computer programming approach was adopted, which fits the entire spectrum relying on a database of the relative intensities that can contribute to PIXE spectra.

The analysis of PIXE spectra with computer program usually comprises two steps. The first step involves determination of the peak area for the principal line of each element present and then, through some standardization technique, the conversion of that area to an elemental concentration. The first step is usually achieved from a least-squares fit of a model to the measured spectrum and this demands knowledge of the detector response or line shape, the background spectrum shape and the calibration of the detector / electronic system. It also requires a data base of relative $X$-ray line intensities together with other physical information pertaining to the 
modification of these by matrix effects. The second step includes many options, ranging from an absolute reliance on the database records to stored data obtained from standards.

\subsubsection{The Guelph PIXE (GUPIXWIN) software}

Several software packages for PIXE spectral analysis have been developed, but the one which is considered to be the most reliable is GUPIX [20]. GUPIXWIN software package has been used to analyze PIXE spectra in the present study. GUPIX is an interactive and versatile software package used for fitting PIXE spectra and deriving elemental concentrations for thin, thick and intermediate layered specimens. GUPIX utilizes the most sophisticated and updated databases for cross sections, stopping powers, attenuation coefficients, etc, and can deal with the analysis of samples of any thickness. The main advantage of this software is that the elemental concentration is extracted from the peak intensities via "fundamental parameters" approach.

\subsubsection{Features of GUPIX Software Package}

Atomic data base: The necessary atomic data of X-ray lines are provided by several subroutine programs in this package. The stopping power coefficients are obtained from the "The Stopping and Ranges of lons in Matter" Pergamon Press, New York by Ziegler, Littmark and Biersack and the X-ray attenuation coefficient values are obtained from the NBS computer code XCOM. The production cross-sections are calculated from the theoretical ECPSSR ionization cross sections. For $\mathrm{K} X$-rays, relative KX-ray intensities are taken from Scofield's data tables, using Bambynek formula the $\mathrm{K}$ shell fluorescence yields calculated and production cross-sections are calculated by using Chen and Crasemann theoretical ECPSSR ionization cross sections,. The $\mathrm{L} X$-ray subshell ionization cross-sections, fluorescence and Coster-Kronig yields and relative intensity ratios used in this software are described by Campbell [20].

Fit elements: The elements whose peaks are in the fit region are referred to as 'fit elements'. The list of elements (including trace elements, matrix elements and sometimes mixture of trace and matrix elements) identified by the user is described as $\mathrm{KX}$ ray elements in the range $Z=6$ to $Z=60, L X$-ray elements in the range $Z=22$ to $Z=92$ and $M X$-ray elements in the range $Z=72$ to $\mathrm{Z}=83$.

Fit Procedure: The standard Marquardt non-linear leastsquare fitting procedure is used to prevent premature elimination of weak peaks. This is followed by an iterated linear square procedure which allows fitting PIXE spectrum containing the X-ray peaks of maximum channel number elements. The line shape of a given X-ray peak is described analytically in terms of channel number. The theoretical spectrum is generated according to the principal component of the peak as a Gaussian or a Voigtian (which is a Gaussian convoluted with the intrinsic $\mathrm{X}$-ray line shape which is Lorentzian) for each of the main X-ray lines (including escape peaks, radiative auger peaks, double ionization satellite peaks and pile-up peaks) of the elements. The constructed spectrum is then filtered by a top-hat digital filter and is compared with the digitally filtered data. The goodness of fit is tested via the chi-square test on the basis of the channel weighing.
Removal of the continuum background: Biological samples subjected to X-ray irradiation exhibit a background continuum. This background is the result of primary charged particles and secondary electron interactions in the sample, and to a lesser extent in the $\mathrm{Si}(\mathrm{Li})$ detector, with the production of bremsstrahlung, Rayleigh, and Compton scatter emissions. Therefore, prior to the quantitative analysis of trace elements in the sample, this background interference must first be removed. This is accomplished with the aid of GUPIXWIN software. In order to remove background "top-hat" digital filter is used. The filtered model spectrum is then fit to the filtered raw data spectrum by using a least-squares procedure. The program decides filter parameters and optimizes them versus energy calibration.

Matrix correction: This software has extensive options for the more difficult problem of determining matrix composition i.e., major element concentrations in thick or intermediate targets and also layer thickness or their concentrations in mutli-layer targets. Using this software package matrix correction can be applied in two ways, depending on whether the matrix is initially defined or undefined. Matrix corrections are applied according to the relative $\mathrm{X}$-ray intensities provided the matrix is defined. In the case of initially undefined matrix, iterative matrix composition and / or layer thickness procedures are used. These procedures include providing information on several aspects such as, the number of layers, beam-target-detector geometry, the incident ion type and energy, etc. A further essential feature is that one can take care of the contributions to matrix effects of the "invisible" elements.

Standardization technique: The elemental concentration can be obtained from peak intensities basing on the good quality PIXE data base and other known aspects of the system (e.g., solid angle, detector thickness etc.), in an absolute fashion i.e., without standards (fundamental parameters approach). Alternatively, another approach can be followed that includes analyses which can be conducted relative to single-element standards or to standard matrices containing various trace elements. The approach followed in GUPIXWIN, called the standardization technique, includes aspects of both the aforementioned approaches, and is based on a single quantity " $\mathrm{H}$ " (solid angle subtended by the front face of the $\mathrm{Si}(\mathrm{Li})$ crystal) that describes the system. In addition, the relative charge collected for each sample is required to convert peak areas into elemental concentrations.

\section{Results and Discussions}

\subsection{PIXE analysis of two biological reference materials}

Peaks of characteristic $X$-rays from elements $\mathrm{K}, \mathrm{Ca}, \mathrm{V}, \mathrm{Mn}$, $\mathrm{Fe}, \mathrm{Ni}, \mathrm{Cu}, \mathrm{Zn}, \mathrm{As}, \mathrm{Se}, \mathrm{Sr}$ and $\mathrm{Pb}$ present in NIST SRM Bovine Liver (1577c) and elements $\mathrm{K}, \mathrm{Ca}, \mathrm{Mn}, \mathrm{Fe}, \mathrm{Cu}, \mathrm{Zn}, \mathrm{Se}, \mathrm{Br}, \mathrm{Rb}$, $\mathrm{Sr}$ and $\mathrm{Pb}$ present in IAEA RM Anima I Blood (A-13) were identified and their concentrations were determined using GUPIXWIN software package. The typical PIXE spectrum of the analyzed NIST SRM and IAEA RM are shown in Fig. 1 and Fig. 2 respectively. The certified and measured concentrations of elements present in both reference materials are shown in 


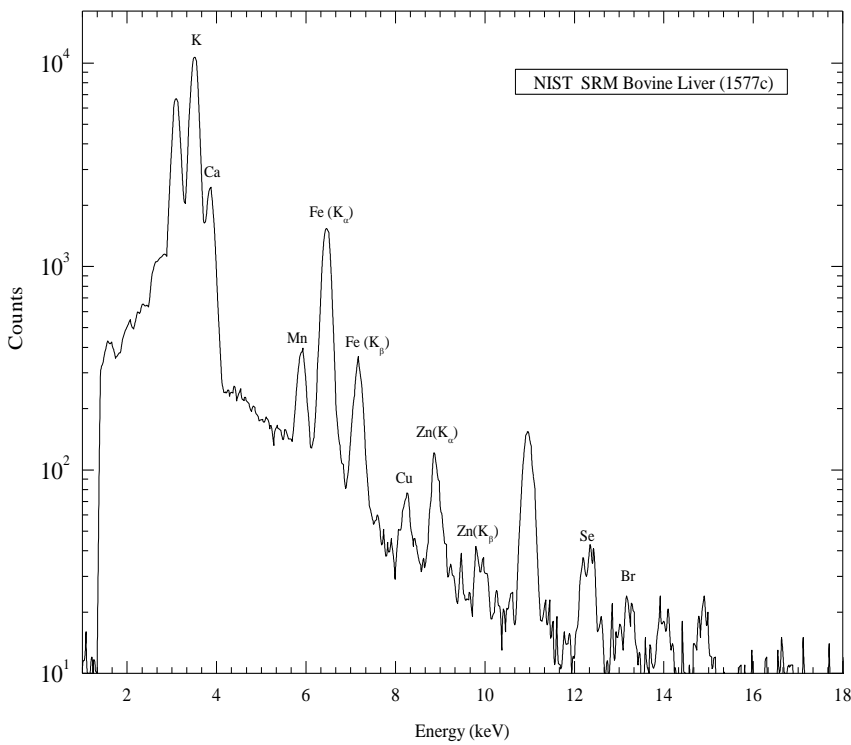

Fig. 1 PIXE spectrum of NIST SRM Bovine Liver (1577c)

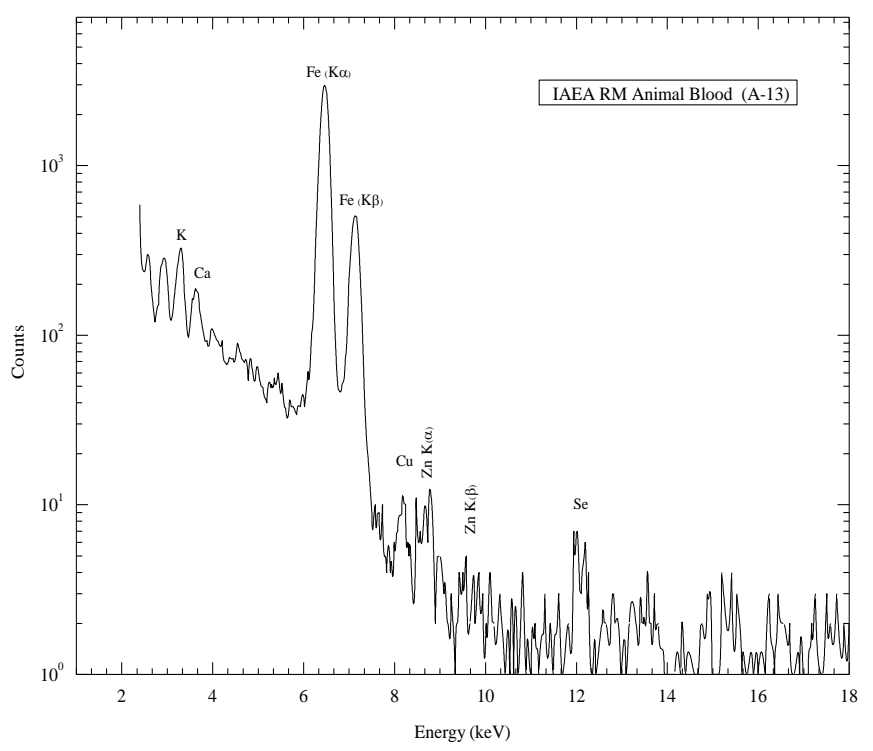

Fig. 2 PIXE spectrum of IAEA RM Animal Blood (A-13)

Table 1

Concentrations (ppm) of trace elements in NIST and IAEA reference materials by PIXE technique

\begin{tabular}{|c|c|c|c|c|}
\hline \multirow{2}{*}{ Element } & \multicolumn{2}{|c|}{ NIST SRM Bovine Liver (1577c) $(\mathbf{n}=\mathbf{5})$} & \multicolumn{2}{c|}{ IAEA RM Animal Blood (A-13) $(\mathbf{n}=5)$} \\
\cline { 2 - 5 } & Certified value & Measured value & Certified value & Measured value \\
\hline $\mathrm{K}$ & $1.023 \pm 0.06(\%)$ & $1.042 \pm 0.07(\%)$ & $2500 \pm 350$ & $2596 \pm 130$ \\
\hline $\mathrm{Ca}$ & $131 \pm 10$ & $141 \pm 14$ & $286 \pm 53$ & $298 \pm 37$ \\
\hline $\mathrm{V}$ & $0.0082 \pm 0.0006$ & $0.0076 \pm 0.0016$ & -- & -- \\
\hline $\mathrm{Mn}$ & $10.46 \pm 0.47$ & $9.26 \pm 0.67$ & $0.05 \pm 0.03$ & $0.08 \pm 0.02$ \\
\hline $\mathrm{Fe}$ & $197.94 \pm 0.65$ & $201 \pm 13$ & $2400 \pm 150$ & $2514 \pm 10$ \\
\hline $\mathrm{Ni}$ & $0.044 \pm 0.009$ & $0.052 \pm 0.008$ & -- & -- \\
\hline $\mathrm{Cu}$ & $275 \pm 4$ & $263 \pm 9$ & $4.3 \pm 0.5$ & $4.64 \pm 0.25$ \\
\hline $\mathrm{Zn}$ & $181 \pm 1$ & $190 \pm 6$ & $13 \pm 1$ & $13.6 \pm 0.7$ \\
\hline $\mathrm{As}$ & $0.0196 \pm 0.0014$ & $0.021 \pm 0.0017$ & -- & -- \\
\hline $\mathrm{Se}$ & $2.03 \pm 0.045$ & $2.14 \pm 0.12$ & $0.24 \pm 0.08$ & $0.27 \pm 0.05$ \\
\hline $\mathrm{Br}$ & -- & -- & $22 \pm 2.5$ & $21.15 \pm 1.5$ \\
\hline $\mathrm{Rb}$ & -- & -- & $2.3 \pm 0.7$ & $2.1 \pm 0.45$ \\
\hline $\mathrm{Sr}$ & $0.095 \pm 0.004$ & $0.097 \pm 0.015$ & $0.69 \pm 0.46$ & $0.85 \pm 0.24$ \\
\hline $\mathrm{Pb}$ & $0.063 \pm 0.001$ & $0.073 \pm 0.016$ & $0.18 \pm 0.08$ & $0.20 \pm 0.03$ \\
\hline
\end{tabular}

Table 1. From Table 1, it is noticed that for the number of samples analyzed for NIST Bovine Liver (1577c), elements with a coefficient of variance (CV) less than $5 \%$ were $\mathrm{Fe}, \mathrm{Cu}$ and $\mathrm{Zn}$, elements with a CV of $5-10 \%$ were $\mathrm{K}, \mathrm{Ca}, \mathrm{Mn}$, As and Se, element with a CV of $10-20 \%$ was $\mathrm{Sr}$ and elements with a CV of $20-25 \%$ were $\mathrm{V}, \mathrm{Ni}$ and $\mathrm{Pb}$. In IAEA Animal Blood, Fe was found to be with a CV less than 1\%, elements with a CV of less than $10 \%$ were $\mathrm{K}, \mathrm{Cu}, \mathrm{Zn}$ and $\mathrm{Br}$, elements with CV of $10-20 \%$ were $\mathrm{Ca}$ and $\mathrm{Pb}$ and elements with $\mathrm{CV}$ of $20-30 \%$ were $\mathrm{Mn}$, Se, $\mathrm{Rb}$ and $\mathrm{Sr}$.

\subsection{Comparison of values determined by PIXE with certified} values

Values determined by PIXE were compared with the certified values of NIST and IAEA as shown in Table 1. The determined elemental concentrations were $88-125 \%$ relative to certified values except for $\mathrm{Mn}$ in Animal Blood samples. In particular, the elemental content of $\mathrm{K}, \mathrm{Ca}, \mathrm{Fe}, \mathrm{Cu}, \mathrm{Zn}, \mathrm{As}$, Se, $\mathrm{Sr}$ were $95-108 \%$ relative to certified values for SRM Bovine
Liver. Agaja and Obiajunwa [21] validated PIXE technique by analysing the SRM Bovine Liver (1577a) and observed 60$100 \%$ of recovery for the elements $\mathrm{K}, \mathrm{Ca}, \mathrm{Mn}, \mathrm{Fe}, \mathrm{Cu}, \mathrm{Zn}, \mathrm{Br}$ and $\mathrm{Rb}$ whereas in the study of Biswas et al.[22], 90-110\% of recovery was reported for the same elements. Campbell [23] reported that the elements $\mathrm{Fe}, \mathrm{Cu}, \mathrm{Mn}, \mathrm{Zn}, \mathrm{Se}, \mathrm{Br}$ and $\mathrm{Rb}$ were within $91-127 \%$ relative to certified values. In this study for the case of IAEA Animal Blood, elements $\mathrm{K}, \mathrm{Ca}, \mathrm{Fe}, \mathrm{Cu}, \mathrm{Zn}, \mathrm{Br}$ and $\mathrm{Rb}$ were obtained with $91-108 \%$ relative to certified values. However, for the same IAEA RM Animal Blood, $87-108 \%$ of recovery was obtained by Kwiatek et al [24] for the elements $P$, $\mathrm{K}, \mathrm{Ca}$ and $\mathrm{Fe}$ and $80-110 \%$ of recovery was reported by Rihway and Halloum [25] for the elements $\mathrm{K}, \mathrm{Ca}, \mathrm{Fe}$ and $\mathrm{Zn}$ relative to certified values.

\section{Conclusion}

The results obtained in the present study reveal that PIXE is a promising analytical tool for analysis of trace elements in different samples particularly those of biological origin. On 
comparing the determined trace elemental content in NIST SRM Bovine Liver (1577c) and IAEA RM Animal Blood (A-13) using PIXE with the certified values, it is found that the reproducibility of PIXE is consistent with NIST and IAEA certified values. It is concluded that PIXE can be used precisely and efficiently in a non-destructive way for simultaneous analysis of several elements.

\section{References}

1. Marguí E, Queralt E, García-Ruiz E, García-González E, Rello $L$ et al. Energy dispersive X-ray fluorescence spectrometry for the direct multi-element analysis of dried blood spots. Spectrochim. Acta B. 2018; 139: 13-19.

2. Wandzilak A, Czyzycki M, Radwanska E, Adamek D, Geraki $K$ et al. X-ray fluorescence study of the concentration of selected trace and minor elements in human brain tumours. Spectrochim. Acta B. 2015; 114: 52-57.

3. Naga Raju GJ, Sarita P, Murthy KSR. Comparative trace elemental analysis of cancerous and non-cancerous tissues of rectal cancer patients using PIXE. Nucl. Instr. Meth. Phys. Res. B. 2017; 404: 146-149.

4. Zaichick V, Zaichick S. INAA application in the assessment of chemical element mass fractions in adult and geriatric prostate glands. Appl. Radiat. Isot. 2014; 90: 62-73.

5. Arain SA, Kazi TG, Afridi HI, Talpur FN, Mughal MA et al. Estimation of copper and iron burden in biological samples of various stages of hepatitis $C$ and liver cirrhosis patients. Biol. Trace. Elem. Res. 2014; 160: 197-205.

6. Afridi, HI, Brabazon D, Kazi TG, Naher S. Evaluation of essential trace and toxic elements in scalp hair samples of smokers and alcohol user hypertensive patients. Biol. Trace Elem. Res. 2011; 143: 1349-1366.

7. Markiewicz B, Sajnóg A, Lorenc W, Hanć A, Komorowicz I et al. Multielemental analysis of 18 essential and toxic elements in amniotic fluid samples by ICP-MS: Full procedure validation and estimation of measurement uncertainty. Talanta. 2017; 174: 22-130.

8. Naga Raju GJ, Sarita P, Ravi Kumar, M, Ramana Murty GAV, Seetharami Reddy B et al. Trace elemental correlation study in malignant and normal breast tissue by PIXE technique. Nucl. Instr. Meth. Phys. Res. B. 2006; 247: 361-367.

9. Naga Raju GJ, Sarita P, Ramana Murty GAV, Ravi Kumar M, Seetharami Reddy B et al. Trace elemental analysis of normal, benign hypertrophic and cancerous tissues of the prostate gland using the particle-induced $X$ ray emission technique. Eur. J. Cancer Prev. 2007;16: 108-115.

10. Chandrasekhar Rao J, Naidu BG, Sarita P, Naga Raju GJ. PIXE and ICP-MS analysis of Andrographis Paniculata Medicinal Plant. Iop Conf. Ser. Mater. Sci. Eng. 2017; 225: 012235.

11. Johansson TB, Akselsson R, Johansson SAE, "X-ray analysis: Elemental trace analysis at the 10-12 g level," Nucl. Instr. Meth. Phys. Res. B. 1970; 84: 141-143.

12. Padhi HC, L-subshell ionization studies with heavy charged particles. Indian J. Phys. 1995; 69: 109-120.

\section{Acknowledgement}

The authors thank the authorities and staff of lon Beam Laboratory, Institute of Physics, Bhubaneswar, for providing the Pelletron Accelerator facility and for rendering technical assistance. One of the authors, P. Sarita, is thankful to UGC, New Delhi for funding this work in the form of a Major Research Project (F.No. 42-831/2013 (SR)).

13. Berger MJ, Hubbell JH. XCOM: Photon cross sections on a personal computer, 1987.

14. Pajek M, Kobzev AP, Sandrick R, lkhamov RA, Khusmurodov $\mathrm{SH}$. Accurate efficiency determination of a $\mathrm{Si}(\mathrm{Li})$ detector in the $\mathrm{Si}-\mathrm{K}$ and $\mathrm{Au}-\mathrm{M}$ absorption edge energy region. Nucl. Instr. Meth. 1989; B22: 346-358.

15. Orlic I, Snow CH, Tang SM. Experimental L-Shell X-Ray Production and Ionization Cross Sections for Proton Impact. At. Data and Nucl. Data Tables. 1994; 56: 159210.

16. Paul H, Sacher J. Fitted empirical reference cross sections for K-shell ionization by protons," At. Data and Nucl. Data Tables. 1989; 42: 105-156.

17. Paul H, Bolik O, Fitted Empirical Reference Cross Sections for K-Shell Ionization by Alpha Particles. At. Data and Nucl. Data Tables. 1993; 54: 75-131.

18. Maxwell, JA, Campbell, JL, Teesdale WJ. The Guelph PIXE software package. Nucl. Instr. Meth. Phys. Res. B. 1989; 43: 218-230.

19. Liu Z, Cipolla S. ISICS: A program for calculating K-, Land M-shell cross sections from ECPSSR theory using a personal computer. Comput. Phys. Commun. 1996; 97; 315-330.

20. Campbell JL, Boyd NI, Grassi N, Bonnick P, Maxwell JA. The Guelph PIXE software package IV. Nucl. Instr. Meth. Phys. Res. B. 2010; 268: 3356-3363.

21. Agaja SA, Obiajunwa El. Proton - Induced X - Ray Emission (PIXE) analysis of some sea food from Western Niger Delta, Nigeria. Am. J. Food. Nutr. 2012; 2(2): 5154.

22. Biswas SK, Khaliquzzaman M, Islam MM, Khan AH. The use of a single multielement standard for trace analysis in biological materials by external beam PIXE. Nucl. Instr. Meth. Phys. Res. B. 1984; 1984: 337-342.

23. Cambell JL. Progress in Biological Applications of PIXE. IEEE Transactions on Nuclear Science. 1979; 26: 13631367.

24. Kwiatek WM, Kubica B, Paluszkiewicz C, Gałka M. Trace element analysis by means of synchrotron radiation, $\mathrm{XRF}$, and PIXE: selection of sample preparation procedure. J. Alloys Compd. 2001; 328: 283-288.

25. Rihawy MS, Halloum D. Characterization and application of in-vacuum PIXE/EBS system for the direct elemental analysis of thick solid biological samples. Appl. Radiat. Isot. 2018; 133: 14-21. 